\title{
A propos de Serpentostephanus Sudarikov, 1961
}

\author{
(Strigeata: Cyathocotyloidea: Prohemistomidae: Szidatinae)
}

\author{
par G. DUBOIS \\ Grand-Rue, 12, CH-2035 Corcelles
}

\section{Résumé.}

Le genre Serpentostephanus Sudarikov, 1961 est inclus dans la sous-famille des Szidatinae Dubois, 1938, dont la diagnose est amendée. Il est à rapprocher de Gogatea Lutz, 1935, Szidatia Dubois, 1938 et Mesostephanoides Dubois, 1951. Une clé de détermination de ces genres est proposée.

\section{Summary.}

About Serpentostephanus Sudarikov, 1961 (Strigeata : Cyathocotyloidea : Prohemistomidae : Szidatinae).

The genus Serpentostephanus Sudarikov, 1961 is placed in the subfamily Szydatinae Dubois, 1938, the diagnosis of which is emended. It is near to being Gogatea Lutz, 1935, Szidatia Dubois, 1938 et Mesostephanoides Dubois, 1951. A key of the genera is proposed.

Le genre Serpentostephanus (1) a été créé par V. E. Sudarikov (1961, pp. 397-398) pour le Prosostephanus natricis que nous avions décrit (1958, pp. 63-65) comme parasite de Natrix maura (L.) en provenance de Gafsa (Tunisie), déposé dans la collection R.-Ph. Dollfus, à Paris (Muséum National d'Histoire Naturelle).

L'attribution de ce Ver au genre Prosostephanus semblait se justifier par le développement relativement grand de l'organe tribocytique et par la position en tandem des testicules. Mais elle constituait une exception notable, en ce sens que les Prosostephaninae Szidat, 1936 sont inféodés aux Mammifères (genres Prosostegénérique.

(1) On peut regretter que Sudarikov n'ait pas choisi la racine grecque pour composer le nom

Accepté le 12 décembre 1978. 
phanus Lutz, 1935 et Tangiella Sudarikov, 1961) et aux Oiseaux (genres Duboisia Szidat, 1936 et Neogogatea Chandler et Rausch, 1947). Sudarikov le maintint dans cette sous-famille.

Chez Serpentostephanus natricis (Dub.), l'organe tribocytique, qui est fixé en protrusion chez les cinq syntypes, ne dépasse pas la ventouse ventrale (située à peine en avant du milieu du corps, plus exactement aux 38-41/100). Les follicules vitellogènes $\mathrm{y}$ font intrusion en se disposant en couronne.

Une condition à peu près semblable a été observée par $\mathrm{R}$. Ph. Dollfus (1953, fig. 2) chez Szidatia nemethi Dollf., de Natrix viperina (Latr.) : l'organe dilaté fait «fortement saillie dans la concavité du segment antérieur, dont il occupe plus de la moitié de la longueur et presque toute la largeur ", sans outrepasser la ventouse ventrale. Par contre, chez Szidatia joyeuxi (Hughes, 1929), du même hôte, il est moins proéminent (donc relativement plus petit), et sa cavité apparaît sous forme d'une fente médiane (Dubois, 1938, fig. 334 ; Dollfus, op. cit., fig. 1) (2).

Une semblable variation s'observe chez Gogatea serpentium (Gogate), où l'organe tribocytique peut être complètement rétracté et creusé d'une cavité (cf. Mehra, 1947, fig. 1 et 2), entouré par deux rangs de follicules disposés en fer à cheval, ou en protrusion, offrant alors l'aspect d'une masse ovale qui renferme la totalité des vitellogènes (cf. Gogate, 1932, fig. 1; Dubois, op. cit., fig. 337 ; Agrawal, 1966, fig. 7) (3).

Ces faits permettent de rapprocher le genre Serpentostephanus Sudar. de Szidatia Dub. et de Gogatea Lutz, et de l'inclure avec ces derniers dans la sousfamille des Szidatinae Dub., 1938, inféodée aux Serpents (4) et dont la diagnose amendée serait la suivante :

\section{Subfamilia Szidatinae Dubois, 1938}

(Syn. Gogatinae Mehra, 1947)

Prohemistomidae à organe tribocytique moyen, ovale ou arrondi, creux à l'état de rétraction (à fente longitudinale), occupant tout ou partie de la seconde moitié du corps (si celui-ci est indivis) ou du segment antérieur (s'il est bisegmenté), selon

(2) Chez les Szidatia, le corps est nettement bisegmenté, et les follicules vitellogènes constituent deux amas longitudinaux, un de chaque côté de l'organe tribocytique.

(3) R. C. Chatterji (1940, fig. 3) a représenté un état intermédiaire de l'organe tribocytique de Gogatea serpentium.

(4) Au contraire, chez les Prosostephaninae Szidat, 1936 (genres Prosostephanus Lutz, 1935, Duboisia Szidat, 1936, Neogogatea Chandler et Rausch, 1947 et Tangiella Sudarikov, 1961), l'organe tribocytique manifeste une tendance à l'hypertrophie, s'allongeant ou s'épaississant pour devenir linguiforme ou massif, sans ouverture, presque aussi large que le corps dont il occupe tout ou partie de la concavité ventrale, dépassant presque toujours la ventouse ventrale faiblement développée ou absente pour atteindre souvent le niveau du pharynx. 
qu'il est en protrusion ou rétracté, la ventouse ventrale étant à mi-longueur ou à peine plus en avant; à follicules vitellogènes contenus dans ces limites et disposés soit en deux amas latéraux et parallèles (Szidatia), soit en couronne ou en fer à cheval (Serpentostephanus, Gogatea, Mesostephanoides), pouvant faire intrusion dans l'organe tribocytique lors de son expansion. Parasites de Reptiles (Serpents : Colubridés).

La sous-famille des Szidatinae comprend quatre genres: Gogatea Lutz, 1935, Szidatia Dubois, 1938, Mesostephanoides Dubois, 1951 et Serpentostephanus Sudarikov, 1961.

\section{Clé de détermination}

1. Follicules vitellogènes formant deux amas longitudinaux, un de chaque côté de l'organe tribocytique. Eufs moyens (jusqu'à $115 \mu$ de long/55-70 $\mu$ ). Tunisie,

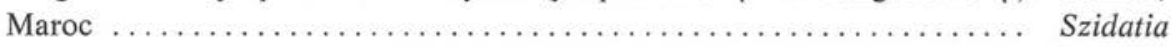

- Follicules vitellogènes groupés en une couronne ou un fer à cheval autour de l'organe tribocytique, pouvant y faire intrusion lors de son expansion. CEufs



2. Corps plus ou moins distinctement bisegmenté, à segment antérieur ovale, concave ventralement, à segment postérieur subcylindrique ou conique, plus court que le précédent. Second testicule toujours situé hors de la couronne vitelline, parfois très en arrière dans le segment postérieur. Inde, Birmanie $\ldots \ldots \ldots \ldots \ldots \ldots$

- Corps non bisegmenté (ou prolongé seulement par un petit appendice caudal), naviculaire, ovale et concave ventralement. Les deux testicules sont inclus dans la couronne vitelline. Tunisie ...................... Serpentostephanus

3. Cirre énorme, pouvant atteindre presque $300 \mu$ de longueur et une trentaine de $\mu$ de diamètre à l'état de protraction. Gonades disparaissant au cours de la maturité sexuelle. Metraterm bien développé, 120-150/20-30 $\mu$. Rapport : longueur du corps / diamètre moyen de la couronne vitelline $>4 \ldots$ Mesostephanoides

- Cirre court et étroit, $45 \mu$ de long, $6 \mu$ de large. Gonades subsistant durant la maturité sexuelle. Metraterm absent ou faiblement développé. Rapport: longueur du corps / diamètre moyen de la couronne vitelline: environ $3 \ldots \ldots$ Gogatea

\section{Bibliographie}

Agrawal V. (1966): Studies on some reptilian trematodes from Lucknow. Indian J. Helminth., 18, 62-76.

Chatterji R. C. (1940): Helminth parasites of the snakes of Burma, I. Trematoda. Philipp. J. Sci. Manila, 71, 381-401. 
Dollfus R.Ph. (1953): Miscellanea helminthologica maroccana VII. Les Szidatia de Natrix viperina (Latreille, 1802) [Trematoda Digenea]. Arch. Inst. Pasteur Maroc, 4, 505-512.

Dubois G. (1938) : Monographie des Strigeida (Trematoda). Mém. Soc. Neuchâtel. Sci. Nat., 6, $1-535$.

Dubois G. (1958): Quelques Strigeida (Trematoda) de la collection R.-Ph. Dollfus. Bull. Soc. Neuchâtel. Sci. Nat., 81, 55-67.

Gogate B.S. (1932): On a new species of trematode (Prohemistomum serpentum n. sp.) from a snake, with a note on an immature species of Heterechinostomum Odhner from the cat. Parasitology, 24, 318-320.

Mehra H. R. (1947): Studies on the family Cyathocotylidae. Part 2. A contribution to our knowledge of the subfamily Prohemistominae Lutz, 1935, with a discussion on the classification of the family. Proc. Nat. Acad. Sci. India, 17, 1-52.

Sudarikov V.E. in Skrjabin K. I. (1961): [Ordo Strigeidida (La Rue, 1926) Sudarikov, 1959. Subordo Cyathocotylata Sudarikov, 1959]. In: [Trematodes of animals and man. Principles of trematodology], 19, 267-415, Moscow. 